ISAHP Article: Mancini, Vacca/ENAV Top 5 Improvement Areas To Be Submitted to the International Symposium of the Analytic Hierarchy Process 2014, Washington D.C., U.S.A.

\title{
ENAV TOP 5 Improvement Areas
}

\author{
Lorenzo Vacca \\ Consorzio Sicta c/o \\ Safety Department - ENAV S.p.A. \\ Rome, Italy \\ E-mail: lorenzo.vacca.sicta@enav.it \\ Maurizio Mancini \\ Head of Risk Assessment Department \\ Safety Department - ENAV S.p.A. \\ Rome, Italy \\ E-mail: maurizio.mancini@enav.it
}

\begin{abstract}
Top 5 IA is a methodology developed by ENAV, the Italian Air Navigation Service Provider. The objective is to identify, from a Safety point of view, the top 5 areas of improvement of Air Traffic Control Units.

One of the Italian most complex (in terms of traffic and layout) Air Traffic Control Units, is the site that has been selected for this study.

The method is based on the analysis of the answers that air traffic controllers gave to an ad-hoc questionnaire. Then, those answers have been evaluated by means of AHP (Analytic Hierarchy Process) methodology.

The weights obtained have been mediated in order to determine a team-representative set of weights which have been combined with all the SME questionnaire answers. The results at last have been statistically analyzed to achieve the Top 5 Improvement Areas representative of the Air Traffic Control Unit.

The activities identified by the model represent the starting point to schedule a set of enhancements and monitoring activities to continuously improve the Safety of the ATC Unit.
\end{abstract}

Keywords: AHP, Air Traffic Control Unit, ATC, ATM, improvement area, pairwise comparison, questionnaire, risk, risk factor, Safety, weights. 


\section{Introduction}

ICAO and Single European Sky regulations have established common requirements for the Safety of air navigation services provision encompassing all aspects related to procedures, human factors, technology and operational environment.

The Safety Objective defined by the "Network strategy plan 2012 - 2019" aims at "establishing the Top 5 pan-European ATM Operational Safety Risks and developing action plans collaboratively with all concerned to reduce risks".

Therefore an ad-hoc study has been structured in order to identify and characterize those complexity factors that controllers working in tower environment face in day-by- day operations. In this way it was possible to determine the five Top Improvement Areas.

\section{Literature Review}

Considering the continuous and dynamic interactions among the three elements of ATM functional system and the correlation with the key concepts of capacity and workload, complexity has been largely studied in many research projects on ATM.

ENAV methodology was essentially based on two sources: EUROCONTROL and FAA. EUROCONTROL developed a study to identify top 5 operational risks of 2013.

The study has been conducted involving several Air Navigation Service Providers and starting from the incidents data and investigation reports.

ENAV has decided to shift its point of view from a reactive to a proactive perspective without directly dealing with incident data but focusing on subject matter expert involvement (i.e. air traffic controllers with a proven operational experience) .

In doing so, ENAV has taken into account a complexity study developed by the FAA, the U.S. Air Navigation Service Provider.

FAA analysis aimed at evaluating the complexity of several airports in USA. The data were collected by means of questionnaires distribution to Air Traffic Controllers.

Each questionnaire was structured into different complexity areas, each complexity area was characterized by several complexity factors.

Air traffic controllers were asked to evaluate each complexity factor by defining its contribution to the Unit complexity and by assessing its likelihood.

ENAV has developed a similar questionnaire structured into several improvement areas and related factors. The interviewees were asked to estimate the impact of every factor on the Safety of operations.

Unlike FAA, ENAV study was enhanced in order to include the AHP methodology.

\section{Hypotheses/Objectives}

Towards service provisioning excellence, ENAV decided to develop a methodology to identify the Top 5 Improvement Areas of its Air Traffic Control Units aiming at supporting a decision making process and effort allocation by means of objective, rational and measurable criteria.

The main objective of TOP 5 IA is to provide a tool for a preliminary identification of the topics that an Air Traffic Control Unit should take into account to improve the Safety level of its operations.

The exploitation of Air Traffic Controllers experience mixed with the rigorous application of AHP methodology represents the added value of this study. 


\section{Research Design/Methodology}

The study was organized as follows:

- identification of two "Macro Areas" (i.e. "Operational Framework" and "Human Performance")

- identification of nine "Risk Areas"

1. airport characteristics,

2. airspace characteristics,

3. traffic characteristics,

4. ATC procedures,

5. equipment,

6. meteo,

7. training,

8. human factor and

9. environmental conditions.

The first six risk areas belong to "Operational Framework" area while the last three belong to "Human Performance" area.

- identification of "Risk Factors" per each "Risk Area"

- creation of a questionnaire (made up of two parts) to be filled out by Air Traffic Controllers in order to evaluate "Risk Factors",

- Application of AHP methodology to evaluate:

1. "Macro Areas",

2. "Risk Areas" (per each macro area)

- statistical analysis of answers.

Figure 1 represents hierarchy identified for the model by means of Superdecisions SW.

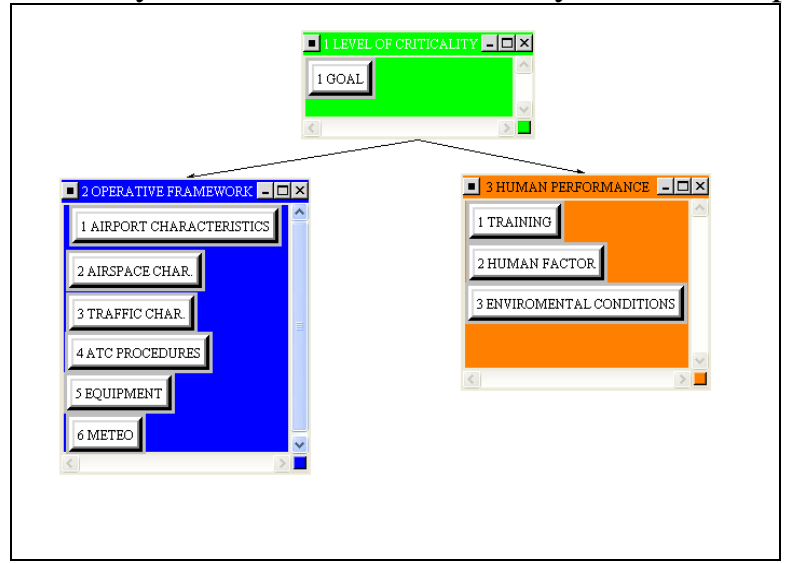

Figure 1 - Model Hierarchy

The questionnaire has been designed in order to include both complexity analysis (Part 1) and pairwise comparison (Part 2).

\section{Part 1 Questionnaire}

Per each "Risk Factor", ATCOs were asked to evaluate how much it could affect the safety of the operations, using a scale from " 1 " (minimum level of risk) to "5" (maximum level of risk). 


\section{Part 2 Questionnaire}

This part was dedicated to the AHP methodology application. ATCos were asked to perform pairwise comparison between "Macro Areas" and between "Risk Areas" (per each "Macro Area".

\section{Analysis of results}

All questionnaires have been analyzed to calculate both "Risk Factor" magnitude and judgment matrices related to the pairwise comparison, and, each one has been revised with the ATCOs in order to tune the "consistency index".

The final output of every judgment matrix was, as well, submitted to the SME validation. All the judgment matrices have been merged using the geometric mean and the resulting matrices have been processed through Superdecision SW to gain the team representative risk areas weights.

The abovementioned weights have been validated by the SME team and, for each questionnaire, the risk magnitude values, associated to "Risk factors", have been derived according to the formula below:

\section{Risk Magnitude Value=Risk factor value *Risk Area Weight*Risk Macro Area Weight}

At last all the risk magnitude values have been statistically analyzed and merged using the tool "StatTools 6" developed by Palisade.

The Top 5 Improvement Areas correspond to the Areas with the 5 higher "Risk Magnitude Values".

\section{Data/Model Analysis}

The analysis have been conducted on a pool of 30 experienced Air Traffic Controllers.

For all judgment matrices, the recorded "Consistency Index" has not never been higher than 0,11324 .

The geometric mean of judgment matrices, their related "Consistency Index" and Local weights are listed below:

\begin{tabular}{|c|c|c|c|c|c|c|c|c|}
\hline \multicolumn{6}{|c|}{ 2. Node comparisons with respect to $1 \mathrm{GOAl}$} & \multicolumn{3}{|c|}{ 3. Results } \\
\hline \multicolumn{6}{|c|}{ Graphical Verbal Matrix Questionnaire Direct. } & \multirow{2}{*}{\multicolumn{3}{|c|}{ Inconsistencyy 0.000793}} \\
\hline \multicolumn{6}{|c|}{$\begin{array}{l}\text { Comparisons wit "1 GOAL" node in "2 AMBIENTE OPERATIVO" cluster } \\
3 \text { CAR. TRAFFICO is } 1.9231 \text { times more important than } 2 \text { CAR.SPAZIO AEREO }\end{array}$} & & & \\
\hline Inconsistency & 2CAR.SPAZ & 3 3 CAR. TRAN & $\frac{\text { Pre Important }}{4 \text { PROCEDUR }}$ & than 2 CAR.S & GMETEO & \begin{tabular}{|l} 
1CAR.AER \\
2CAR SPA
\end{tabular} & & 0.32559 \\
\hline 1 CAR.AERO & $\leftarrow \leftarrow 5.7899$ & $\leftarrow 3.2700$ & $\leftarrow 2.16$ & $\leftarrow 1.22$ & $\leftarrow 2.2599$ & 3CAR. TR & & 0.12398 \\
\hline 2 CAR.SPAZ & & $\uparrow 19230$ & $\uparrow 3.125$ & 1 33333 & $\uparrow 1.8867$ & 4 PROCEDU & & 0.16866 \\
\hline 3 CAR. TRAN & & & \begin{tabular}{|l|l|l}
$\uparrow 1333$ \\
\end{tabular} & $\uparrow 1.2820$ & $\leftarrow 1.04$ & 6 METEO & & 0.13063 \\
\hline 4 PROCEDUR & & & & $\uparrow 1.0526$ & $\leftarrow 1.28$ & & & \\
\hline 5EQUPMEN & & & & & $\leftarrow 1.26$ & & & \\
\hline
\end{tabular}

Figure 2 - Judgment Matrix \& Local Weights/C.I. - Operative Framework

\begin{tabular}{|c|c|c|c|c|}
\hline \multicolumn{3}{|c|}{ 2. Node comparisons with respect to $1 \mathrm{GOAL}$} & \multicolumn{2}{|c|}{ 3. Results } \\
\hline \multicolumn{3}{|c|}{ Graphical Verbal Matrix Questionnaire Direct } & Normal - & Hybrid - \\
\hline \multirow{2}{*}{\multicolumn{3}{|c|}{$\begin{array}{l}\text { Comparisons wit "1 GOAL" node in "3 COMPONENTE UMANA" cluster } \\
\text { 2FATTORI UMANI is } 1.2346 \text { times more important than } 1 \text { TRAINING }\end{array}$}} & \multicolumn{2}{|c|}{ Inconsistency: 0.00001} \\
\hline & & & 1 TRAINING & 0.32206 \\
\hline Inconsistency & 2FATTORI & 3 CONDIZ, & 2FATTORI & 0.39856 \\
\hline 1 TRAINING & \multirow[t]{2}{*}{$\uparrow \longdiv { 1 . 2 3 4 5 }$} & $\leftarrow 1.15$ & $3 \mathrm{CONDIZ} \sim$ & 0.27938 \\
\hline 2FATTORI & & 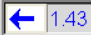 & & \\
\hline
\end{tabular}

Figure 3 - Judgment Matrix \& Local Weights/C.I. - Human Performance

International Symposium of the Analytic Hierarchy

Process June 29 - July 2, 2014 
As shown in the pictures above the final C.I. are better that the ones obtained in the individual analysis.

The weights gained have been used to calculate the abovementioned "Risk Magnitude Values" and have been validated by all the ATCOs involved.

\section{Limitations}

The model proposed can surely be improved acting on two main features:

1. The AHP judgment matrices could be merged using a different and more structured approach rather than using the geometric mean method;

2. There is not the assurance of having covered all the operational aspect of the Air Traffic Management System - the questionnaire could be surely optimized during the further analysis that are going to be performed on the other Air Traffic Control Units.

\section{Conclusions}

The present study has led to the definition of an instrument capable of combining the operational experience of the Air Traffic Controllers with the features of the AHP methodology in order to identify the Improvement Areas by a Safety perspective.

The activities acknowledged represent the starting point to schedule a set of enhancements and monitoring activities to continuously improve the Safety of the ATC Units.

Another strength of this methodology is represented by its simplicity and by its minimal impact on the effort of the SME involved.

Even if the methodology is relatively simple to perform, the results obtained are remarkable because they are fully able to summarize and represent the ATCos perception of the operational environment.

Anyway the methodology could be improved by means of acting on an optimized approach for the group decision making process and by means of sharpening the topics contemplated by the questionnaire.

\section{Key References}

- Koros, Della Rocco, Panjwani, Ingurgio, D’Arcy (2003). Complexity in Air Traffic Control Towers: A Field Study. U.S. Department of Transportation (FAA).

- EUROCONTROL (2013). Top 5 ATM Operational Safety Priorities for 2013. EUROCONTROL.

- T.Saaty (1982). Decision Making for Leaders: The Analytical Hierarchy Process for Decisions in a Complex World. Wadsworth. 1988. 